Pacific Journal of Mathematics

A CHARACTERIZATION OF SCALAR TYPE OPERATORS ON

EarL Rob rt Berks 


\title{
A CHARACTERIZATION OF SCALAR TYPE OPERATORS ON REFLEXIVE BANACH SPACES
}

\author{
EARL BERKSON
}

Introduction. The main purpose of this paper is to characterize scalar operators on reflexive Banach spaces. This is accomplished in 4.2 and 4.4. However, most of the results are not limited to reflexive spaces.

We give a fundamental decomposition theorem for scalar operators in $\S 2$, and show in $\S 3$ that this decomposition is unique.

In what follows, all spaces are over the complex field, all Banach algebras have an identity of norm 1, and an operator will be a bounded linear transformation with range contained in its domain. This understanding will also cover material quoted from other sources.

1. Preliminaries. In this section we reproduce some machinery from [4] and [7] which will be needed in the sequel.

The definitions and results of this paragraph are taken from [4].

Definition. Let $X$ be a vector space. A semi-inner-product on $X$ is a mapping [,] of $X \times X$ into the field of complex numbers such that:

(i) $[x+y, z]=[x, z]+[y, z]$ for $x, y, z \in X$.

(ii) $[\lambda x, y]=\lambda[x, y]$ for $x, y \in X, \lambda$ complex.

(iii) $[x, x]>0$ for $x \neq 0$.

(iv) $|[x, y]|^{2} \leqq[x, x][y, y]$.

We then call $X$ a semi-inner-product space (abbreviated s.i.p.s). If $X$ is a s.i.p.s., then $[x, x]^{1 / 2}$ is a norm on $X$. On the other hand, every normed linear space can be made into a s.i.p.s. (in general, in infinitely many ways) so that the semi-innner-product is consistent with the norm-i.e., $[x, x]^{1 / 2}=\|x\|$, for each $x \in X$. By virtue of the HahnBanach theorem this can be accomplished by choosing for each $x \in X$ exactly one bounded linear functional $f_{x}$ such that $\left\|f_{x}\right\|=\|x\|$ and $f_{x}(x)=\|x\|^{2}$, and then setting $[x, y]=f_{y}(x)$, for arbitrary $x, \mathrm{y} \in X$.

Definition. Given a linear transformation $T$ on a s.i.p.s., we denote by $W(T)$ the set, $\{[T x, x] \mid[x, x]=1\}$, and call this set the numerical range of $T$.

An important fact concerning the notion of numerical range is the following:

Received July 16, 1962. 
1.1 Let $X$ be a Banch space and $T$ an operator on $X$. Although in principle there may be many different semi-inner-products consistent with the norm of $X$, nonetheless if the numerical range of $T$ relative to one such semi-inner-product is real, then the numerical range relative to any such semi-inner-product is real. If this is the case, we call $T$ a hermitian operator.

Also important is the result:

1.2 If $X$ is a s.i.p.s., complete with respect to the induced norm on $X$, and $T$ is a linear transformation on $X$, bounded with respect. to the induced norm, then $|W(T)| \leqq\|T\| \leqq 4|W(T)|$, where $|W(T)|$ denotes the quantity $\sup \{|\lambda| \mid \lambda \in W(T)\}$.

In [7], I. Vidav introduces the following notion of hermiticity:

Definition. An element $h$ of a Banach algebra $A$ with identity $e$ will be called hermitian if and only if for $\alpha$ real, $\|e+i \alpha h\|=$ $1+o(\alpha)$ as $\alpha \rightarrow 0$.

It is shown in $[4 ; \S 9]$ that an operator $T$ on a Banach space $X$ is a hermitian operator if and only if it is hermitian in the sense of Vidav's definition-i.e., if and only if $\|I+i \alpha T\|=1+o(\alpha)$ for $\alpha$ real, where $I$ is the identity operator. Thus we have at our disposal two equivalent formulations of the notion of hermiticity for operators on Banach spaces.

The result of [7] very important for our considerations is the following:

1.3 Suppose $A$ is a Banach algebra with identity $e$. Let $H$ be the set of hermitian elements of $A$ (i.e., $H=\{h \in A \mid\|e+i \alpha h\|=$ $1+o(\alpha)$, for $\alpha$ real $\})$. We assume that: (a) every $a \in A$ has a representation $a=u+i v, u, v \in H$; (b) if $h \in H$, then there is a representation $h^{2}=u+i v$ such that $u, v \in H$ and $u v=v u$. Then there is an involution on $A$ and a new norm equivalent to the given norm such that in terms of this involution and the new norm $A$ is a $C^{*}$-algebra. It is well known that the Gelfand representation of a commutative $C^{*}$-algebra with identity is an isometry onto $C(\mathscr{C})$, the algebra of all continuous complex-valued functions on the maximal ideal space $\mathscr{M}$ (see, for example, [3; $§ 26 \mathrm{~A}]$ ). Hence we can state:

1.4 Let $A$ be a commutative Banach algebra with identity, and let $H$ be the set of hermitian elements of $A$. If every $a \in A$ has a representation in the form $a=u+i v$, where $u, v \in H$, then the Gelfand representation of $A$ is a bicontinuous isomorphism of $A$ onto $C(\mathscr{H})$.

2. A fundamental decomposition. Throughout the rest of this paper $X$ will be a fixed Banace space with norm \|\| , and $X^{*}$ will be its dual. Throughout this section $S$ will be a fixed scalar operator 
on $X$, and $E$ will be its resolution of the identity (see [1] for this terminology). For given $x \in X, x^{*} \in X^{*}$, we shall denote by var $x^{*} E(\quad) x$ the quantity $\sup \Sigma\left|x^{*} E\left(\sigma_{i}\right) x\right|$, where the supremum is taken over all finite sequences $\left\{\sigma_{i}\right\}$ of disjoint Borel sets in the complex plane $p$.

It is shown in [1; see proof of Theorem 17] that there is a constant $K$ such that

$$
\operatorname{var} x^{*} E(\quad) x \leqq K\|x\|\left\|x^{*}\right\|, x \in X, x^{*} \in X^{*} .
$$

We now show:

2.2 Lemma. For each $x \in X$, define $|x|$ by

$$
|x|=\sup _{\substack{x^{*} \in X^{*} \\\left|\| x^{*}\right| \mid=1}} \operatorname{var} x^{*} E(\quad) x
$$

Then | | is a norm on X equivalent to || ||.

Proof. It is straightforward to verify that || is a seminorm. With $K$ as in 2.1 we have that $|x| \leqq K\|x\|$, for $x \in X$. Given $x \in X$, choose $x^{*} \in X^{*}$ so that $\left\|x^{*}\right\|=1$ and $x^{*}(x)=\|x\|$. Then $\|x\|=$ $\left|x^{*}(x)\right|=\left|x^{*} E(p) x\right| \leqq|x|$. This completes the proof.

2.3 Lemma. Relative to the norm | | defined in 2.2, $E(\sigma)$ is a hermitian operator, for each Borel set $\sigma$.

Proof. We shall also use the symbol $\mid$ to denote the norm of an operator relative to || . We shall show that if $\sigma$ is a Borel set, and $E(\sigma) \neq 0$, then

2.4

$$
|I+i \alpha E(\sigma)|=|1+i \alpha| \text {, for } \alpha \text { real . }
$$

For arbitrary $x \in X, x^{*} \in X^{*}$, with $\left\|x^{*}\right\|=1$, real $\alpha$, and arbitrary finite sequence $\sigma_{1}, \sigma_{2}, \cdots, \sigma_{n}$ of disjoint Borel sets, we have:

$$
\begin{aligned}
& \sum_{j=1}^{n}\left|x^{*} E\left(\sigma_{\jmath}\right)(I+i \alpha E(\sigma)) x\right| \\
& =\sum_{j=1}^{n}\left|x^{*} E\left(\sigma_{j}\right)\left[E\left(\sigma^{\prime}\right) x+(1+i \alpha) E(\sigma) x\right]\right|, \\
& \quad \text { where } \sigma^{\prime} \text { is the complement of } \sigma . \\
& \leqq \sum_{j=1}^{n}\left|x^{*} E\left(\sigma_{j}\right) E\left(\sigma^{\prime}\right) x\right|+|1+i \alpha| \sum_{j=1}^{n}\left|x^{*} E\left(\sigma_{j}\right) E(\sigma) x\right| \\
& \leqq|1+i \alpha| \operatorname{var} x^{*} E(\quad) x, \quad \text { since the sets } \sigma_{1} \cap \sigma^{\prime}, \cdots, \sigma_{n} \cap \sigma^{\prime}, \\
& \leqq \\
& \sigma_{1} \cap \sigma, \cdots, \sigma_{n} \cap \sigma \text { are disjoint. }
\end{aligned}
$$




\section{Hence}

$$
|(I+i \alpha E(\sigma)) x| \leqq|1+i \alpha||x|
$$

So

$$
|I+i \alpha E(\sigma)| \leqq|1+i \alpha|
$$

On the other hand, if $y$ is in the range of $E(\sigma)$, with $|y|=1$, then $|(I+i \alpha E(\sigma)) y|=|y+i \alpha y|=|1+i \alpha|$. Thus 2.4 is established. Since $|1+i \alpha|=1+o(\alpha)$, the desired conclusion follows.

2.5 THEOREM. There are operators $R$ and $J$ such that:

(1) $S=R+i J$.

(2) $R J=J R$.

(3) Relative to some norm on $X$ equivalent to \|\|$, R^{m} J^{n}$ are hermitian operators for $m, n=0,1,2, \cdots$.

Proof. We write

2.6 $S=\int_{\text {"real part of" } \operatorname{Re} \lambda d E(\lambda)+i \int \operatorname{Im} \lambda d E(\lambda) \text {, where "Re" and "Im" denote }}$ We now set $R=\int \operatorname{Re} \lambda d E(\lambda)$ and $J=\int \operatorname{Im} \lambda d E(\lambda)$. Clearly (1) and (2) hold. For the proof of (3) we use the norm | |, as defined in 2.2. By (2.3), $E(\sigma)$ is a hermitian operator relative to | |, for each Borel set $\sigma$. It is clear from the definition in 1.1 that a sum of real multiples of hermitian operators is a hermitian operator, and so is a limit in the uniform operator topology of hermitian operators. The conclusion in (3) is now clear from the fact that for arbitrary positive integers $m, n$,

$$
\begin{aligned}
R^{m} & =\int(\operatorname{Re} \lambda)^{m} d E(\lambda) \\
J^{n} & =\int(\operatorname{Im} \lambda)^{n} d E(\lambda) \\
R^{m} J^{n} & =\int(\operatorname{Re} \lambda)^{m}(\operatorname{Im} \lambda)^{n} d E(\lambda) .
\end{aligned}
$$

REMARK 1. It is not known, in general, if a product of commuting hermitian operators is hermitian, or even if the powers of a hermitian operator are hermitian. Consequently it is not known if part of property (3) of 2.5 is superflous. ${ }^{1}$

REMARK 2. We shall show in $\S 3$ that the decomposition described

1 See note added in proofreading. 
in 2.5 is unique. Thus the representation of $S$ given by 2.6 is characterized by properties (1), (2), and (3) of 2.5. In [2; §5], Foguel has introduced the representation 2.6 , but has characterized it differently, without the notion of hermitian operator. In accordance with his terminology, we call $\int \operatorname{Re} \lambda d E(\lambda)$ and $\int \operatorname{Im} \lambda d E(\lambda)$ the real and imaginary parts of $S$, respectively.

3. Uniqueness of the decomposition in 2.5. In this section we show that the decomposition given in the statement of 2.5 is unique. At first glance it might seem that uniqueness is immediate from 1.2; however, given two pairs of operators, each pair satisfying (1)-(3) of 2.5, we do not assume that the norms given in (3) are the same for the two pairs.

Some additional items will be needed. Given an element $a$ of a Banach algebra $A$, we shall denote by $s p_{A}(a)$ the spectrum of $a$ in $A$. We shall denote by $[X]$ the Banach algebra of all operators mapping $X$ into itself. We shall use the fact that if $x$ is a hermitian element of the Banach algebra $A$, then $s p_{A}(x)$ is real. This is shown in [7; Lemma 2].

3.1 TheOREm. Let $R$ and $J$ be any two operators on $X$ satisfying conditions (2) and (3) of 2.5, and let $A$ be the Banach subalgebra of $[X]$ generated by $R, J$, and $I$. Further, let $T=R+i J$, and define the functions $f_{1}$ and $f_{2}$ on $s p_{[X]}(T)$ by

$$
f_{1}(\lambda)=\operatorname{Re} \lambda, \quad f_{2}(\lambda)=\operatorname{Im} \lambda .
$$

Then there is a bicontinuous isomorphism of $C\left(s p_{[x]}(T)\right)$ onto $A$ such that the image of $f_{1}$ is $R$ and the image of $f_{2}$ is $J$.

Proof. We shall assume throughout the proof that $X$ has been renormed with an equivalent norm, |||||| , chosen according to condition (3) of 2.5, and likewise that $[X]$ and $A$ have been renormed with the corresponding operator norm, which we also denote by \|\|$\quad \|$. We shall also introduce a semi-inner-product on $X$ (denoted by $[$,$] )$ consistent with $\|\mid\|$. We first show that $A$ satisfies the hypotheses of 1.4. It is clear that if $Q \in A$, then $Q$ is the limit in the uniform operator topology of a sequence $\left\{P_{n}\right\}$ of polynomials in $R$ and $J$ with complex coefficients. For each $n, P_{n}$ can be written in the form $P_{n}=U_{n}+i V_{n}$. where $U_{n}$ and $V_{n}$ are polynomials in $R$ and $J$ with real coefficients. Thus $U_{n}$ and $V_{n}$ belong to $A$ and are hermitian operators on $X$. For arbitrary positive integers $m$ and $n$, and for arbitrary $x \in X$ with $\|x\|=1$,

$$
\left|\left[\left(U_{m}-U_{n}\right) x, x\right]+i\left[\left(V_{m}-V_{n}\right) x, x\right]\right|=\left|\left[\left(P_{m}-P_{n}\right) x, x\right]\right| \leqq||\left|P_{m}-P_{n}\right||| .
$$


Since $\left[\left(U_{m}-U_{n}\right) x, x\right]$ and $\left[\left(V_{m}-V_{n}\right) x, x\right]$ are real,

$$
\left|\left[\left(U_{m}-U_{n}\right) x, x\right]\right| \leqq||\left|P_{m}-P_{n}\right||| \text { and }\left|\left[\left(V_{m}-V_{n}\right) x, x\right]\right| \leqq||\left|P_{m}-P_{n} \|\right| \text {. }
$$

Hence by 1.2 ,

$$
\text { || } U_{m}-U_{n}|||\leqq 4||| P_{m}-P_{n}|| \mid \text { and }|| V_{m}-V_{n}|| \leqq 4||\left|P_{m}-P_{n} \|\right| \text {. }
$$

It follows that $\left\{U_{n}\right\}$ and $\left\{V_{n}\right\}$ converge to hermitian operators $U$ and $V$, respectively, which belong to $A$. Therefore $Q=U+i V$. Since $U$ and $V$ are hermitian operators lying in $A$, they are (by $\S 1$, paragraph 3) hermitian elements of the algebra $A$. Thus 1.4 holds.

To complete the proof we show that there is a one-to-one mapping $\psi$ of $\mathscr{C}$, the maximal ideal space of $A$, onto $s p_{[X]}(T)$ such that if $\hat{R}$ and $\hat{J}$ denote the Gelfand representatives of $R$ and $J$, respectively, then $\hat{R}\left(\psi^{-1}(\lambda)\right)=R e \lambda$ and $\hat{J}\left(\psi^{-1}(\lambda)\right)=\operatorname{Im} \lambda$, for each $\lambda \in s p_{[X]}(T)$. To accomplish this, we identify $\mathscr{C l}$ with the space of all homomorphisms of $A$ onto the complex numbers. We then define $\psi$ as follows:

3.2

$$
\psi(h)=h(R)+i h(J) \text {, for each homomorphism } h \in \mathscr{M} .
$$

Since $s p_{A}(R)$ and $s p_{A}(J)$ are real, and since $R, J$, and $I$ generate $A$, it is clear that $\psi$ is one-to-one. The range of $\psi$ is obviously the range of the Gelfand representative of $T$, and hence is $s p_{A}(T)$. Since the Gelfand representation of $A$ is a one-to-one map of $A$ onto $C(\mathscr{C})$, it is clear that the commutative Banach algebra $A$ is semi-simple and completely regular. Hence (see [6; Corollary (3.7.6)]), $s p_{A}(T)=s p_{[x]}(T)$. The desired conclusions about $\hat{R}$ and $\hat{J}$ are obvious by virtue of 3.2.

3.3 THEOREM. Let $S$ be a scalar operator on $X$. The operators $R$ and $J$ of 2.5 are uniquely determined by (1)-(3).

Proof. Let $R$ and $J$ satisfy (1)-(3) of 2.5. Then (in the notation of 3.1) there is a bicontinuous isomorphism $\phi$ of $C\left(s p_{[X]}(S)\right)$ onto $A$ such that $\phi\left(f_{1}\right)=R$ and $\phi\left(f_{2}\right)=J$. Let $\mathscr{B}$ be the class of Borel sets in $s p_{[X]}(S)$. By [1; Theorem 18], the adjoint of every operator in $A$ is a scalar type operator of class $X$, and there is a spectral measure $G$ in $X^{*}$ of class $(\mathscr{B}, X)$ such that

3.4

$$
\phi(f)^{*}=\int_{s p[X](S)} f(\lambda) d G(\lambda) \text {, for each } f \in C\left(s p_{[X]}(S)\right) .
$$

In particular, $S^{*}=\int_{s_{[X]}(S)} \lambda d G(\lambda)$. By [1; Lemma 6], the resolution of the identity for $S^{*}$ (call the resolution of the identity $F$ ) is given by

$$
F(\sigma)=G\left(s p_{[X]}(S) \cap \sigma\right)
$$


Thus, using 3.4, we have

$$
\begin{aligned}
& R^{*}=\int_{s_{[X]}(S)} \operatorname{Re} \lambda d F(\lambda) \text { and } J^{*}=\int_{s p_{[X]}(S)} \operatorname{Im} \lambda d F(\lambda), \\
& \text { where } F \text { is the resolution of the identity for } S^{*} .
\end{aligned}
$$

So the adjoints of $R$ and $J$ are uniquely determined, and hence so are $R$ and $J$.

4. Characterization of scalar type operators on reflexive spaces. The first theorem of this section is the converse of 2.5 under the additional hypothesis that $X$ is reflexive. The second theorem contains a summary of preceding results for the special case when $X$ is reflexive.

4.1 THEOREM. Let $X$ be reflexive, and let $T$ be an operator on $X$. Then $T$ is a scalar type operator of class $X^{*}$ if there exist operators $R$ and $J$ satisfying

(1) $T=R+i J$.

(2) $R J=J R$.

(3) Relative to some norm on $X$ equivalent to \|\|$, R^{m} J^{n}$ are hermitian operators for $m, n=0,1,2, \cdots$.

Proof. By 3.1 the Banach subalgebra of $[X]$ generated by $R, J$, and $I$ is equivalent to $C\left(s p_{[x]}(T)\right)$. The desired conclusion is now immediate from [1; conclusion (iv) of Theorem 18], which states that if an algebra of operators on a reflexive Banach space $Y$ is equivalent to the algebra of all continuous complex-valued functions on some compact Hausdorff space, then this algebra of operators consists entirely of scalar type operators of class $Y^{*}$.

REMARK 3. It is a consequence of 2.5 and 4.1 that a spectral operator on a reflexive Banach space $Y$ is automatically of class $Y^{*}$. This is also easy to see directly, since, in the reflexive case, it follows from the Hahn-Banach theorem that a total linear manifold in $Y^{*}$ is dense in the norm topology of $Y^{*}$. Thus for a reflexive space $Y$, the terms "spectral operator" and "spectral operator of class $Y^{*}$ ", are equivalent.

4.2 THeOREM. Let $X$ be reflexive, and let $T$ be an operator on $X$. Then $T$ is a scalar type operator if and only if there exist operators $R$ and $J$ satisfying conditions (1)-(3) of 4.1 If this is the case, $R$ and $J$ are uniquely determined.

It is desirable to replace condition (3) occurring above by a con- 
dition which involves only the original norm on $X$ rather than some equivalent norm. The author wishes to express his appreciation to G. Lumer for communicating to him the essence of the next theorem, which accomplishes this purpose.

4.3 THEOREM. Let $X$ be an arbitrary Banach space with norm \| \|, and let $R$ and $J$ be commuting operators on $X$. Further, let $\mathscr{A}$ be the set of all polynomials in $R$ and $J$ with real coefficients. In order that there exist a norm on $X$ equivalent to \|\| and relative to which $R^{m} J^{n}$ are hermitian operators for $m, n=0,1,2, \cdots$, it is necessary and sufficient that the set $\left\{e^{i P} \mid P \in \mathscr{A}\right\}$ be a bounded subset of $[X]$.

Proof. Suppose that $X$ can be renormed with an equivalent norm, III III, relative to which the operators $R^{m} J^{n}$ are hermitian. Then each $P \in \mathscr{A}$ is clearly a hermitian operator relative to $\|\mid\|$, and so by [7; Lemma 1] $\left\|e^{i P}\right\|=1$. Since the renorming is an equivalent one, $\left\{\left\|e^{i P}\right\| \mid P \in \mathscr{A}\right\}$ is bounded.

Conversely, suppose that the positive number $K$ is an upper bound for $\left\{\left\|e^{i P}\right\| \mid P \in \mathscr{A}\right\}$. Define $\|\mid\|$ on $X$ as follows:

$$
\|x\|=\sup _{P \in \mathscr{A}}\left\|e^{i P} x\right\| .
$$

Clearly $\|x\| \mid\|K\| x \|$. Also $\|x\|=\left\|e^{-i P} e^{i P} x\right\| \leqq K\|x\|$. Since \|\|$\quad \|$ is obviously a seminorm, we can conclude that it is a norm equivalent to \|\| . For arbitrary $Q \in \mathscr{A}$ and arbitrary $x \in X$, we have:

$$
\left\|e^{i Q} x\right\|=\sup _{P \in \mathscr{A}}\left\|e^{i(P+Q)} x\right\| .
$$

Since $\mathscr{A}$ is obviously a real algebra, it is clear that as $P$ ranges through $\mathscr{A}$, so does $P+Q$. Hence $\left\|e^{i Q} x\right\|=\|\| x \|$, and each operator $e^{i Q}$, for $Q \in \mathscr{A}$, is an isometry relative to \|\|$\|$. Thus if $Q \in \mathscr{A}$, the operators $e^{i t Q}$, for real $t$, form a one-parameter group of isometries (relative to $\||\||)$. Since the families $\left\{e^{i t Q}\right\}$ and $\left\{e^{-i t Q}\right\}$, with $t>0$, are (in particular) semi-groups of contraction operators, we have by [5; Theorem 3.1] that the generators $i Q$ and $-i Q$ are dissipative; hence $Q$ is a hermitian operator relative to \|\|$\|$. In particular, each operator of the form $R^{m} J^{n}$, where $m$ and $n$ are nonnegative integers, belongs to $\mathscr{A}$ and so is a hermitian operator relative to $\|\mid\|$.

Using 4.2 and 4.3 , we have:

4.4 Theorem. Let $X$ be reflexive, and let $T$ be an operator on $X$. Then $T$ is a scalar type operator if and only if there exist operators $R$ and $J$ such that: 
(1) $T=R+i J$

(2) $R J=J R$.

(3) If $\mathscr{A}$ denotes the set of all polynomials in $R$ and $J$ with real coefficients, then $\left\{e^{i P} \mid P \in \mathscr{A}\right\}$ is a bounded subset of $[X]$. If this is the case, $R$ and $J$ are uniquely determined.

Note Added in Proofreading. G. Lumer has recently shown that the powers of a hermitian operator are not in general all hermitian, even on a reflexive space. This and other matters of interest to readers of this paper will be found in his forth-coming paper, Spectral operators, hermitian operators, and bounded groups, to appear in Acta Sci. Math., (Szeged).

\section{REFERENCES}

1. N., Dunford, Srectral operators, Pacific J. Math., 4 (1954), 321-354.

2. S. R. Foguel, The relations between a spectral operator and its scalar part, Pacific J. Math., 8 (1958), 51-65.

3. L. H. Loomis, An introduction to abstract harmonic analysis, New York, Van Nostrand, 1953.

4. G. Lumer, Semi-inner-product spaces, Trans. Amer. Math. Soc., 100 (1961), 29-43.

5. G. Lumer and R. S. Phillips, Dissipative operators in a Banach space, Pacific J. Math., 11 (1961), 679-698.

6. C. E. Rickart, General theory of Banach algebras, New York, Van Nostrand, 1960.

7. I. Vidav, Eine metrische Kennzeichnung der selbstadjungierten Operatoren, Math. Zeitschr., 66 (1956), 121-128.

UNiversity of CALIFornia at Los ANGeles 



\title{
PACIFIC JOURNAL OF MATHEMATICS
}

\author{
EDITORS
}

\author{
Ralph S. Phillips \\ Stanford University \\ Stanford, California \\ M. G. Arsove \\ University of Washington \\ Seattle 5 , Washington
}

\author{
J. DugunduI \\ University of Southern California \\ Los Angeles 7, California
}

Lowell J. Paige

University of California

Los Angeles 24, California

\section{ASSOCIATE EDITORS}

E. F. BECKENBACH

T. M. CHERRY
D. DERRY
M. OHTSUKA

H. L. ROYDEN

E. SPANIER
E. G. STRAUS

F. WOLF

\section{SUPPORTING INSTITUTIONS}

UNIVERSITY OF BRITISH COLUMBIA CALIFORNIA INSTITUTE OF TECHNOLOGY UNIVERSITY OF CALIFORNIA MONTANA STATE UNIVERSITY UNIVERSITY OF NEVADA NEW MEXICO STATE UNIVERSITY OREGON STATE UNIVERSITY UNIVERSITY OF OREGON OSAKA UNIVERSITY UNIVERSITY OF SOUTHERN CALIFORNIA
STANFORD UNIVERSITY

UNIVERSITY OF TOKYO

UNIVERSITY OF UTAH

WASHINGTON STATE UNIVERSITY

UNIVERSITY OF WASHINGTON

AMERICAN MATHEMATICAL SOCIETY CALIFORNIA RESEARCH CORPORATION SPACE TECHNOLOGY LABORATORIES NAVAL ORDNANCE TEST STATION

Mathematical papers intended for publication in the Pacific Journal of Mathematics should be typewritten (double spaced), and the author should keep a complete copy. Manuscripts may be sent to any one of the four editors. All other communications to the editors should be addressed to the managing editor, L. J. Paige at the University of California, Los Angeles 24, California.

50 reprints per author of each article are furnished free of charge; additional copies may be obtained at cost in multiples of 50 .

The Pacific Journal of Mathematics is published quarterly, in March, June, September, and December. Effective with Volume 13 the price per volume (4 numbers) is $\$ 18.00$; single issues, $\$ 5.00$. Special price for current issues to individual faculty members of supporting institutions and to individual members of the American Mathematical Society: $\$ 8.00$ per volume; single issues \$2.50. Back numbers are available.

Subscriptions, orders for back numbers, and changes of address should be sent to Pacific Journal of Mathematics, 103 Highland Boulevard, Berkeley 8, California.

Printed at Kokusai Bunken Insatsusha (International Academic Printing Co., Ltd.), No. 6, 2-chome, Fujimi-cho, Chiyoda-ku, Tokyo, Japan.

PUBLISHED BY PACIFIC JOURNAL OF MATHEMATICS, A NON-PROFIT CORPORATION

The Supporting Institutions listed above contribute to the cost of publication of this Journal, but they are not owners or publishers and have no responsibility for its content or policies. 


\section{Pacific Journal of Mathematics}

\section{Vol. 13, No. 2 \\ April, 1963}

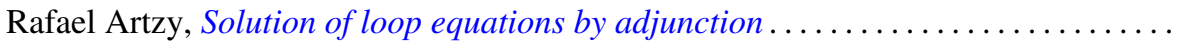

Earl Robert Berkson, A characterization of scalar type operators on reflexive

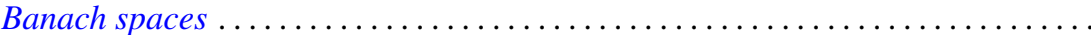

Mario Borelli, Divisorial varieties

365

Raj Chandra Bose, Strongly regular graphs, partial geometries and partially

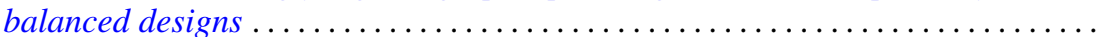

389

R. H. Bruck, Finite nets. II. Uniqueness and imbedding ............... 421

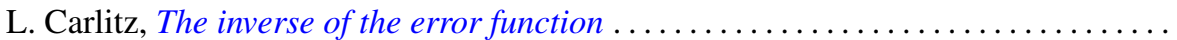

Robert Wayne Carroll, Some degenerate Cauchy problems with operator

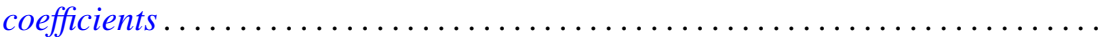

Michael P. Drazin and Emilie Virginia Haynsworth, A theorem on matrices of 0 's

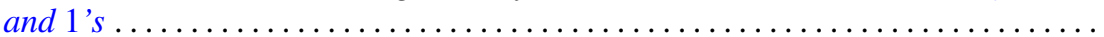

Lawrence Carl Eggan and Eugene A. Maier, On complex approximation .......... James Michael Gardner Fell, Weak containment and Kronecker products of group

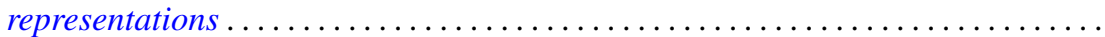

Paul Chase Fife, Schauder estimates under incomplete Hölder continuity

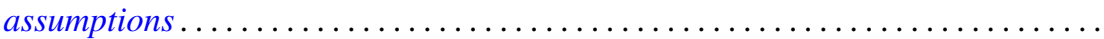

Shaul Foguel, Powers of a contraction in Hilbert space ...................

Neal Eugene Foland, The structure of the orbits and their limit sets in continuous

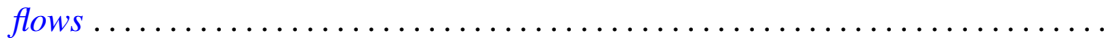

Frank John Forelli, Jr., Analytic measures . . . . . . . . . . . . . . . . . . . . . 563

Robert William Gilmer, Jr., On a classical theorem of Noether in ideal theory ....... P. R. Halmos and Jack E. McLaughlin, Partial isometries .

Albert Emerson Hurd, Maximum modulus algebras and local approximation in

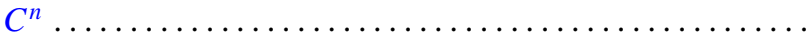

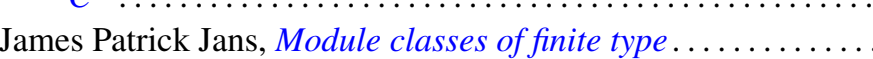

Betty Kvarda, On densities of sets of lattice points ...

H. Larcher, A geometric characterization for a class of discontinuous groups of linear fractional transformations .

John W. Moon and Leo Moser, Simple paths on polyhedra .

T. S. Motzkin and Ernst Gabor Straus, Representation of a point of a set as sum of

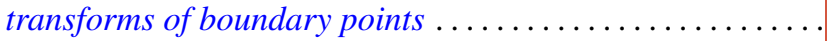

Rajakularaman Ponnuswami Pakshirajan, An analogue of Kolmogorov's three-series theorem for abstract random variables ...............

Robert Ralph Phelps, Čebyšev subspaces of finite codimension in $C(X)$...

James Dolan Reid, On subgroups of an Abelian group maximal disjoint from a given subgroup ...

William T. Reid, Riccati matrix differential equations and non-oscillation criteria for associated linear differential systems .................

Georg Johann Rieger, Some theorems on prime ideals in algebraic number fields ...

Gene Fuerst Rose and Joseph Silbert Ullian, Approximations of functions on the

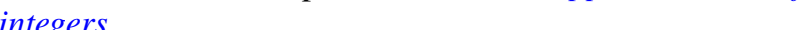

F. J. Sansone, Combinatorial functions and regressive isols . . . . . . . . . . 703

Leo Sario, On locally meromorphic functions with single-valued moduli . . . . . . . 709

Takayuki Tamura, Semigroups and their subsemigroup lattices.

Pui-kei Wong, Existence and asymptotic behavior of proper solutions of a class of second-order nonlinear differential equations . . ........... 